\title{
SIPHON FLOW ACROSS THE MAGNETIC NEUTRAL-LINE OF AN ACTIVE REGION
}

\author{
S.K. SOLANKI \& I. RÜEDI \\ Institute of Astronomy, ETH-Zentrum, CH-8092 Zürich, Switzerland \\ D. RABIN \\ National Solar Observatory, NOAO^, P.O. Box 26732, Tucson, AZ 85726, USA
}

\begin{abstract}
We present accurate measurements, based on the $g=3$ line at $1.5648 \mu \mathrm{m}$, of the field strength and longitudinal velocity in the vicinity of the magnetic neutral-line of a solar active region. We find that very close to the neutral line the field strength and the flow velocity are correlated in the sense that the magnetic polarity with the larger field strength is associated with a downflow, while the polarity with the weaker field shows an upflow. This is exactly the signal predicted by theoretical models of siphon flows, and constitutes the first reliable detection of a siphon flow on the sun.
\end{abstract}

Keywords: Solar magnetic fields - active regions - siphon flows - infrared polarimetry

\section{INTRODUCTION}

The physical description of siphon flows in loop-shaped solar magnetic flux tubes has been well studied (e.g. Meyer \& Schmidt 1968, Cargill \& Priest 1980, 1982, Thomas 1988, Montesinos \& Thomas 1989, Thomas \& Montesinos 1990, 1991 and Degenhardt $1989,1991)$, but their definite observational confirmation has so far remained elusive. In the present paper we discuss measurements - magnetic field and line-of-sight velocity in a plage area - that are most naturally interpreted as a siphon flow between regions of stronger and weaker magnetic field.

\section{OBSERVATIONS AND MODELLING TECHNIQUES}

The observations were obtained with the east auxiliary of the McMath telescope, the main spectrograph and a $58 \times 62 \mathrm{InSb}$ array detector in an active region located very close to the centre of the solar disk $(\mu=\cos \theta=0.998)$. The slit was oriented approximately $\mathrm{E}-\mathrm{W}$ and crossed the neutral line. Stokes $I \pm V$ spectra of Fe I $15648.5 \AA$, a pure Zeeman triplet with a Landé factor $g=3$, and Fe $I 15652.9 \AA$, with an effective Landé factor $g_{e f}=1.53$, were obtained, from which Stokes $V$ (net circular polarization) was extracted. More information on the data and their reduction is given by Rabin (1991).

We have co-added 3 consecutive spectra to improve the $S / N$ ratio without significant loss of spatial resolution. The resulting 21 spectra are plotted in Fig. 1a. They have been vertically shifted for clarity. The location of the neutral line close to the

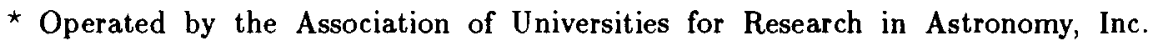
(AURA) under cooperative agreement with the National Science Foundation. 
position of profile No. 8 in Fig. la, coupled with the limited spatial resolution of the observations, leads to the anomalously shaped Stokes $V$ profiles in spectra No. 6-10.

In order to derive information from the spectra, the $V$ profiles of both lines are numerically calculated in a thin-tube magnetic model along multiple rays (so-called 1.5-D radiative transfer). Before comparison with the observed spectra, the synthetic $V$ profiles are spatially averaged to the angular resolution of the observations and spectrally convolved with a Gaussian profile having a Doppler width of approximately $6 \mathrm{~km} \mathrm{~s}^{-1}$ to account for both instrumental smearing and macroturbulent velocity.


Fig. 1 a. Stokes $V / I_{c}$ spectra at different positions along the slit; $I_{c}$ is the local continuum intensity. Each displayed profile is the sum of 3 neighbouring profiles on the original frame. Each spectrum is offset by $2.55^{\prime \prime}$ on the solar surface with respect to its neighbours along the spectrograph slit. The coadded spectra are numbered at the left of the frame. b. Variation of the field strength $B$ along the slit. c. Line-of-sight flow velocities. 


\section{RESULTS}

The fits of the synthetic to the observed $V$ profiles of both lines yield the field strengths $B$ at the height $z=0$ (corresponding to $\tau_{5000}=1$ in the quiet sun) and velocity shifts shown in Figs $1 \mathrm{~b}$ and $1 \mathrm{c}$, respectively. The $B(z=0)$ and velocity values derived for each spectrum are plotted at the same level as the zero-level of that spectrum in Fig. 1a. Positive polarity fields are plotted dashed in Fig. $1 \mathrm{~b}$; negative polarity fields are plotted solid. Positive velocities in Fig. 1c are directed away from the observer, i.e. mainly downwards in the solar atmosphere (only the line-of-sight velocity component is measured). The origin of the velocity scale is determined by requiring that the velocity must disappear far from the neutral line, in accordance with older Stokes observations (e.g. Stenflo \& Harvey 1985, Solanki 1986).

A good fit to most of the spectra is obtained by employing only one magnetic flux-tube component. However, near the neutral line two flux-tube components are necessary to reproduce individual observed profiles. These complex profiles, the fits to them and the synthetic $V$ profiles of the 2 individual magnetic components are plotted in Fig. 2.


Fig. 2a. Stokes $V / I_{c}$ profiles of Fe I $15648.5 \AA$ at the 5 spatial positions requiring two magnetic (and one non-magnetic) components to fit the profiles (spectra are labelled). Solid curves: measured solar spectra, dashed curves: synthetic profiles. b. Synthetic $V / I_{c}$ profiles of the individual magnetic components forming the composite synthetic profiles shown in Fig. 2a. 
Comparing Fig. 1b with Fig. 1c we see the following behaviour near the neutral line: the weaker-field (negative polarity) component exhibits an apparent upflow, while the stronger-field (positive polarity) component shows a downflow.

\section{DISCUSSION}

Matter is flowing downwards in the positive polarity flux tubes with stronger fields and upwards in the weaker fields of the negative polarity flux tubes. Let us make the natural assumption that opposite polarity flux tubes situated so close to the neutral line are connected by magnetic loops. Because the curved flux tube forming the loop obeys lateral pressure balance, the footpoint with weaker field (lower magnetic pressure) has higher gas pressure; while the opposite is true for the other footpoint. Thus, the observed flow satisfies the conditions required of a siphon flow (e.g. Thomas and Montesinos 1991): an upflow in the loop footpoints with higher gas pressure (weaker field) and a downflow in the loop footpoints of lower gas pressure (stronger field).

The advantages of observing siphon flows in the infrared are clear. Due to the larger Zeeman sensitivity in the infrared it is easier to accurately determine the field strength in each magnetic component, which must be known in order to reliably identify a siphon flow. In addition, for spatially unresolved flows the Stokes $V$ profile shape of a strongly split line is more sensitive to the detailed field strengths and flow velocities.

We plan to continue investigating siphon flows along two main avenues: a) We hope to determine how common siphon flows are and if they are related to the development of the magnetic field near neutral lines (e.g. are they related to flares?). Further 1.56 $\mu \mathrm{m}$ spectra on a 2-D grid of the solar surface containing a neutral line have already been obtained. To enhance the diagnostics, Stokes $Q$ and $U$ have been measured in addition to $V$. b) We also aim to determine the physical conditions in the flux tubes supporting the observed siphon flow (are shocks present?). (1992).

A more detailed descripton of the present investigation is given by Rüedi et al.

\section{REFERENCES}

Cargill, P.J., Priest, E.R., 1980, Solar Phys. 65, 251

Cargill, P.J., Priest, E.R., 1982, Geophys. Astrophys. Fluid Dyn. 20, 227

Degenhardt, D., 1989, Astr. Ap. 222, 297

Degenhardt, D., 1991, Astr. Ap. 248, 637

Harvey, J.W., 1985, in Measurements of Solar Vector Magnetic Fields, M.J. Hagyard

(Ed.), NASA Conf. Publ. 2374, p. 109

Meyer, F., Schmidt, H.U., 1968, Zs. Angew. Math. Mech. 48, 218

Montesinos, B., Thomas, J.H., 1989, Ap. J. 337, 977

Rabin, D., 1991, Ap. J. 391, 832 in press

Rüedi, I., Solanki, S.K., Rabin, D., 1992, Astr. Ap. 261, L21

Solanki, S.K., 1986, Astr. Ap. 168, 311

Stenflo, J.O., Harvey, J.W., 1985, Solar Phys. 95, 99

Thomas, J.H., 1988, Ap. J. 333, 407

Thomas, J.H., Montesinos, B., 1990, Ap. J. 359, 550

Thomas, J.H., Montesinos, B., 1991, Ap. J. 375, 404 